\title{
La prise en compte de l'action collective dans l'enseignement et la recherche en management
}

\author{
Yves Hallée \\ Université du Québec à Chicoutimi
}

Dans un contexte de flexibilité marqué par le changement, plusieurs auteurs sont d'avis que le groupe remplace graduellement l'individu comme unité de production (Jacob, 1992, Lapointe, 1995, Roy, 1999). On évoque notamment le fait que les besoins d'efficacité et d'efficience concourent à la création de collectifs de travail plus adapté (Roy, 1999). Les bouleversements associés à la concurrence, l'ouverture des frontières, les développements technologiques et techniques de production «exigent des niveaux de flexibilité, de rapidité, d'efficacité et d'efficience du personnel qui ne peuvent être
L'organisation serait davantage un construit collectif qu'une conséquence externe ou une volonté stratégique soit intégrée au travail (Hammer et Champy, 1993). L'organisation évoluerait donc vers un rapprochement temporel et opérationnel de la décision et de l'exécution, jadis séparation fondatrice de la démarche taylorienne (Lorino, 2007). Ces exemples témoigneraient aussi d'une rupture prononcée avec l'école de la prise de décision et un des auteurs les plus marquants de la pensée du management contemporain, Herbert Simon. Selon Aktouf (2006:193), « [1]'individu [y était considéré comme] un maillon de la chaîne dont la fonction est de véhiculer les informations vers les sommets qui vont traiter, choisir et obtenus que par l'utilisation d'équipes polyvalentes relativement autonomes » (Roy, 1999 :78). À cela se greffent les tentatives contemporaines de rapprocher la prise de décision de l'action, caractérisées notamment par les équipes de travail semi-autonomes et les équipes de projet où des fonctions décisionnelles sont assumées. Ce couplage prise de décision et action particularise même la réingénierie des processus lorsque l'on suggère comme mesure opérationnelle d'accroissement de la valeur ajoutée, qui se traduit par une réduction des coûts et du temps associé aux activités, que la prise de décision décider ». À notre avis, ces changements renforcent l'idée que l'entreprise demeure et a toujours été un espace de vie commune, à destin économique partagé, qui est ment et non le résultat d'actions ou d'activités répondant à un plan conçu d'avance. L'organisation serait davantage un construit collectif qu'une conséquence externe ou une volonté stratégique. C'est ce que nous tenterons de démontrer. À noter que ce texte cherche à susciter la réflexion sur la pertinence d'introduire, en mana-gement, une véritable théorie de l'action collective. nécessairement défini et structuré collective-

\section{Le paradigme rationaliste en management et le modèle de prise de décision}

Les sciences du management sont encore fortement imprégnées par ce paradigme rationaliste centré sur le décideur rationnel et stratège (Aktouf, 2006). Elles sont aussi fondées sur «l'organisation-lieu de processus décisionnels rationnels »(Lorino, $2007: 70)$. Le décideur y est perçu comme le dépositaire du savoir, ce qui légitime toutes ses intentions. Comme les personnes sont supposées être rationnelles et feront ce qui est dans leur 
intérêt, le gestionnaire s'attend à ce que les salariés adoptent sa vision lorsqu'il invoque une série d'arguments sur le bien-fondé de ses orientations. On cherche à convaincre ; et une fois convaincus, les salariés passeraient naturellement de l'orientation à l'acte (Collerette, Delisle et al., 2002). Ainsi, l'organisation est maintes fois représentée comme un objet contrôlable, observable de l'extérieur et largement manipulable à des fins opératoires par les décideurs (Lorino, 2007). La quête ultime réside dans la légitimation d'un modèle de développement qui se veut rationnel. Même le facteur humain, issu de l'école des relations humaines ${ }^{1}$, est passé sous le joug des dirigeants. D'innombrables «mesures manipulatrices ont transformé les résultats originels en recettes » (Aktouf, 2006 : 178-9). Si le travailleur ne veut pas lui-même devenir rationnel et raisonnable, la science ainsi modélisée offrira une partie des moyens pour l'amener malgré lui à ces résultats.

Cette perspective présuppose que l'acteur, le décideur, est considéré comme le point de départ, celui par lequel tout débute. L'action dériverait de l'exécution de plans conçus à l'avance «où l'agir humain relève a priori d'une volonté subjective indépendante, orientée vers des fins individuelles clairement définies»(Joas, 1999: 19). Ainsi, «l'acteur instrumente $[\ldots]$ autrui comme un environnement objectif, selon un protocole élaboré en son for intérieur» (Gislain, $206: 247)$. Cette théorie de l'action rationnelle, dominante en management, est ainsi réduite à une théorie de la planification où l'acteur décide des actions à réaliser par un raisonnement stratégique procédant des fins aux moyens. L'individu est appréhendé de façon isolée et sans référence au réel en action (Friedman et McDaniel, 1998). Ce dernier est exagérément considéré comme «l'atome logique » de l'analyse puisqu'il constituerait l'élément premier de tout phénomène social ${ }^{2}$. Max Weber (1975), dans son étude de l'acteur, c'est-à-dire d'une action rationnelle en valeur (par conviction) ou d'une action rationnelle en finalité (utilisation de stratégies et tactiques pour parvenir à telle ou telle fin), entre dans ce cadre analytique. La théorie des jeux et ses différentes déclinaisons sont également considérées à la différence que le choix et l'action résultent d'interactions stratégiques entre plusieurs agents où l'on cherche notamment à découvrir la stratégie, la convention, la logique inhérente à l'action humaine (Ramstad, 1994).

\section{L'individu est appréhendé de façon isolée et sans référence au réel en action}

\section{Le déterminisme et le fonctionnalisme en management}

Le management se caractérise aussi par une pensée déterministe et fonctionnaliste (Aktouf, 2006 :77). Elle est déterministe dans la mesure où l'évolution de l'organisation ne serait attribuée qu'à l'adaptation à des déterminants extérieurs et fonctionnaliste puisqu'il suffit, pour la pérennité de l'entreprise, de chercher les dysfonctionnements du «mécanisme », considérant qu'elle évoluerait selon une fin invariable ou inévitable. Or, une collectivité ne fonctionne pas selon une fin précise et connue où chacun doit faire, sans autre possibilité (Becker, 2002 : 254). «Rien ne détermine un résultat final, ni dans son occurrence ni dans sa forme » (ibid., : 111). Un mécanisme «naturel » ne connaît ni la rareté, ni les besoins, ni la surpopulation, ni les ressources limitées, ni l'acte d'économiser et ni la futurité (Commons, 1934 :635).

L'organisation est souvent représentée comme différentes fonctions faisant partie d'un ensemble d'éléments différentiés, interdépendants et ordonnés qui concourt à l'atteinte de résultats dans un environnement donné. Une telle approche vise à gérer les fonctions 
de l'entreprise en symbiose avec les autres et en tant qu'éléments d'un univers plus grand, c'est-à-dire, celui de l'organisation en interaction avec son environnement. Cette vision systémique (théorie des systèmes ${ }^{3}$ ) de la réalité organisationnelle inciterait les décideurs à concevoir les différentes fonctions comme un sous-système cohérent d'activités qui contribuent à l'équilibre et à l'atteinte des objectifs organisationnels. En d'autres termes, cette perspective présente les différentes fonctions organisationnelles comme faisant partie d'un tout cohérent et comme un élément en lien avec les stratégies et les objectifs organisationnels qui découlent de l'analyse de l'environnement externe de l'organisation.

Il y aurait certes lieu de se méfier de cette forme de domination, d'autoritarisme environnemental, inéluctable, fataliste, au point de considérer l'organisation comme une entité organique et biologique pour qui l'adaptation à l'environnement devient la condition de survie. Même si l'environnement influence, cette explication n'est pas complète. Qui plus est, elle ne dit pas ce qu'il faut faire (Bernoux, 2004). Selon un registre davantage économique, Gislain et Steiner (1995: 31) soulignent 1'acuité de l'apport de Thorstein Veblen $^{4}$ lorsqu'il critique la conception déterministe de l'action humaine. Comme ces derniers précisent, l'homo æeconomicus est un atome qui n'a «ni antécédent, ni conséquent. Il est un donné humain, isolé et définitif, en équilibre stable sauf lorsque des forces externes le déplacent dans une direction ou une autre ». Cette théorie moléculaire de la société, dérivée des sciences physiques et chimiques, conçoit la société comme une somme de molécules, dont la finalité découle des forces aveugles de la nature, tel le mouvement engendré par les vagues d'un océan, plutôt que comme un ensemble de citoyens avec des droits, des devoirs et des responsabilités réciproques (Commons, 1934 : 119). D'ailleurs, les inspirateurs de l'économie orthodoxe, dont Bentham et Smith, ont souvent cherché à appliquer les modèles rationnels connus des sciences physiques, chimiques et biologiques à la réalité humaine afin d'en ressortir des lois universelles de comportements plus faciles à expliquer et à démontrer (ibid. : 216, 228, 238). Des auteurs plus contemporains professant la même doctrine, tel Friedman (1953), se sont intéressés à la métaphore de la sélection naturelle de Darwin en cherchant à démontrer que la compétition économique entre firmes pour le profit est analogue à la compétition entre les organismes pour 1'existence ${ }^{5}$ (Ramstad, 1994 :77).

Une perspective largement similaire mais davantage présente dans la littérature sociologique et explicative du comportement individuel mérite d'être soulignée. Il s'agit de la théorie de l'action structurelle dans laquelle les déterminations sociales conditionneraient l'action des acteurs. L'explication du comportement individuel se retrouverait ainsi à un niveau plus macro, comme une sorte de conditionnement social. La théorie de l'action de Parsons (1937) sous-tend que la société intervient dans le façonnement des orientations subjectives et des motivations de ses membres par le processus d'intériorisation des valeurs et normes morales comme déterminant de l'action (déterminisme normatif) (Joas, 1999). Pour Bourdieu (1994: 45) et son concept d'habitus, les sujets sont le produit de l'incorporation de structures objectives et de schèmes d'action qui orientent la perception de la situation et la réponse qui est adaptée. Ce processus d'intériorisation des normes sociales conditionne l'agir des individus. Au niveau organisationnel, cela reviendrait à dire que les actions individuelles seraient entièrement déterminées par les structures. Bien que ces dernières contraignent l'individu à travers les relations de pouvoir de la direction, cela n'enlève pas les marges de manœuvre et d'autonomie des individus et des groupes (Bernoux, 2004 : 53). 


\section{Et que dires des interactionnistes ?}

Les situations organisationnelles sont aussi relationnelles. Une perspective largement mésestimée des analyses en management, surtout nord-américaines, est celle des interactionnistes. Elle implique une pluralité d'acteurs à interprétations multiples des actions en cours. Les interactionnistes offrent une perspective centrée sur un réseau de relations d'échange et de règles complexes.

Selon cette approche, «l'interaction a le statut de facteur explicatif central, car il est impossible de com-

\section{Les interactionnistes offrent une perspective centrée sur un réseau de relations d'échange et de règles complexes}

source du pouvoir, c'est-à-dire la notion clé de la dynamique du pouvoir. Reprenant la perspective de Crozier et Friedberg, Donnadieu (2005:233) précise que le «pouvoir dépend du degré d'imprévisibilité que [l'individu saura] maintenir à l'égard des autres; moins ils peuvent prévoir ce [qu'il décide] de faire et plus [il aura] du pouvoir sur eux ». On imagine les stratégies de pouvoir en négociation et l'utilisation de rapports de force, de même que le maintien des zones d'incertitude en cas de prendre les faits sociaux en excluant les relations entre les acteurs et leur influence réciproque » (Bernoux, 2004 : 11). Les normes de comportement résulteraient de l'interaction entre les acteurs. L'entreprise est présentée comme un réseau d'interactions composé d'acteurs stratégiques qui poursuivent des objectifs différents, sinon contradictoires, et qui s'ajustent en permanence selon le dualisme conflit et coopération. Ces acteurs intelligents, donc dotés de mémoire, de projets et de capacités d'anticipation, ne s'impliquent pas sans raison dans une relation sociale et cherchent avant toute chose à maximiser leur utilité (Donnadieu, 2005 : 229-30). Dans cette approche, on cherche à comprendre les enjeux, les jeux de pouvoir, les règles du jeu, les alliances et les stratégies des acteurs.

Crozier et Friedberg (1981:386) estiment que les individus sont prêts à changer dans la mesure où ils sont capables d'y trouver leur intérêt. L'élément décisif du comportement, selon ces auteurs, réside dans le jeu de pouvoir et d'influence auquel l'individu participe et dans lequel il affirme son existence sociale. L'acteur fera donc obstacle à tout ce qui menace son autonomie et cherchera à orienter le changement afin de maintenir les zones d'incertitude qu'il contrôle (ibid.). L'incertitude se révèle, dans ce cadre, la menace de grève, de menace de fermeture ou de délocalisation.

L'incomplétude de cette approche, croyonsnous, réside dans le fait que le collectif est limité aux intérêts individuels et stratégiques des acteurs. Il serait ainsi réduit à la somme des intérêts individuels, calculateurs et utilitaires «dans un contexte d'interdépendance stratégique » (Amblard, Bernoux et al., 2005: 41 citant Friedberg, $1993: 15$ ). Chacun y poursuivrait son propre intérêt sans effet corrélatif et réciproque de l'action. Il n'y a pas d'action collective véritable détachée d'une présomption d'utilité stratégique des actions initiées par les acteurs. Les comportements sociaux résultent d'acteurs qui agissent intentionnellement en vue de fins choisies par ces derniers et qui utilisent des stratégies pour les atteindre (Bernoux, 1990).

Dans cette perspective, on fait aussi état de la centralité du sens que l'individu donne à son action, lequel il faut explorer et dont il faut rendre compte (Bernoux, 2004 : 25). Dans un contexte d'entreprise, le sens donné aux règles est établi par la direction. La volonté d'obéir des salariés serait liée au statut du donneur d'ordre et à la croyance envers ces compétences. Bernoux ajoute que l'acceptation du changement et du système est liée à 
son caractère rationnel. On revient là au caractère rationnel lié à la représentation du décideur qui est propre au management. De plus, la référence à Weber est explicite lorsqu'il mentionne que la rationalisation est une caractéristique essentielle du capitalisme, où «la société moderne est fondée sur des actions [...] rationnelles par rapport à ses buts (Bernoux, 2004: 41). Sa position est cependant plus nuancée en regard de l'individualisme méthodologique propre à l'économie orthodoxe dans la mesure où il considère «qu'il faut prendre en compte les relations sociales concrètes et le contexte social dans lesquels les individus sont impliqués »(Bernoux, 2004 : 26). Les règles comme contraintes sociales et comme référence pour dire comment les choses doivent être, sans valeur prescriptive et normative, se combineraient avec le sens donné par les individus (Bernoux, 2004 : 3238). Malgré ces assises rationalistes, cette perspective a au moins le mérite de s'attarder à l'interaction collective dans l'établissement de normes, contrairement au management orthodoxe où l'on semble totalement ignorer les interactions entre individus qui composent l'organisation.
En somme et de façon très sommaire, on comprend qu'en management, l'homme serait un être de raison et aurait seulement besoin de comprendre les raisons de l'ordre pour changer. Mais ce n'est pas si simple. James G. March (1981), auteur reconnu en management, remet en cause la rationalité dans les organisations lorsqu'il met l'accent sur le caractère chaotique des entreprises et la difficulté de lier l'intention initiale au changement réel. Pour Commons (1934 :683) «[c]e n'est pas l'état rationnel de la société qui détermine l'action, c'est un ensemble complexe et merveilleusement irrationnel d'attentes et d'anticipations entre participations qui se confrontent en situation de transaction $^{6}$ ». En d'autres termes, la réalité est loin d'être statique et universelle; elle est évolutive et changeante au gré des intervenants qui $\mathrm{y}$ interagissent. La prochaine section explicitera plus en détail cette théorie de l'action collective issue de l'institutionnaliste pragmatique de John R. Commons, en débutant par certains facteurs inspirateurs de la proposition de départ tirés de la littérature en management.

\section{Vers une théorisation de l'action collective en management}

Selon Lorino (2007:77), les auteurs Henry Mintzberg et James March «se demandent si la théorie des organisations ne devrait pas viser à une théorisation de l'activité collective plutôt qu'à une théorisation de la décision, l'organisation apparaissant comme un système d'actions coopératives et une création dialogique continue ». De surcroît, il précise que «[1]es sciences de la gestion ont [...] besoin d'une théorie de l'activité collective [...] [qui rend] compte de l'activité collective comme processus de construction continu d'une intelligibilité collective» (ibid.). Évidemment, l'acteur individuel ne disparaît pas pour autant du processus décisionnel. Son rôle se modifie de déterminant causal a priori de l'action à une ressource dans l'action (Lorino, 2007).

Henry Mintzberg (1994:111-113-114) soulignait notamment qu'une stratégie n'est pas un processus isolé mais coopératif et peut émerger de l'action collective, sans nécessairement relever d'une intention consciente et délibérée. La haute direction y jouerait non pas le, mais un rôle crucial dans son développement. De même, son élaboration (strategy making) découlerait aussi de l'apprentissage qui résulte de l'expérimentation en situation de travail. "We think in 
order to act, to be sure, but we also act in order to think» (ibid. : 111). Quant à March (1981 :575), il énonçait clairement la nécessité d'une théorie permettant de comprendre le changement dans les organisations.

Les actes individuels sont impliqués dans des actes sociaux plus larges qui dépassent l'individu en faisant intervenir tous les autres membres du groupe. Il en est ainsi car «le tout (la société) est antérieur à la partie (l'individu) et non la partie au tout; la partie s'explique par la référence au tout, et non le tout par référence à la partie» (Mead, 1963:6). La relation est ainsi inversée du groupe vers l'individu. Le travail d'un gestionnaire n'est donc pas un acte individuel, isolé et étranger de la dynamique collective et, exempt de l'interaction permanente, du rapport nécessaire au réel. Dans le monde du travail, les sujets agissants ne sont pas des êtres vraiment autonomes (Gislain, 2003); ils sont en fait largement interdépendants. Il est donc impératif de se débarrasser des modèles du leader visionnaire où prend forme et se termine l'action. Les organisations sont davantage un système de relations où les règles de l'action collective (les règles opérantes de conduite selon la terminologie de Commons) se définissent et se redéfinissent, et sont en perpétuelles mouvances. Pour Becker (1985 : 205-206) cité par Gislain (2006 : 239), l'action collective est l'ajustement réciproque des perspectives d'action de personnes agissant ensemble.

John Dewey, philosophe pragmatisme, disait que la vérité n'est pas quelque chose de passivement atteint, mais la conséquence d'une démarche active, créative, de la part d'individus qui font partie de la réalité. Tout programme doit être intrinsèquement interactif, révisable du fait des expérimentations continuelles, adaptables pour, et par les acteurs locaux. C'est à toutes les parties concernées, qui connaissent la situation, de décider des règles de leur activité commune sur la base de l'expérience qui permet de connaître les faits qui doivent être pris en compte (Bazzoli 1999: 171 citant Commons, 1919: 176-177). Pour le pragmatisme, il n'y a pas de réalité transcendante coupée de l'expérience vécue de l'individu (Renault, 2006). Évidemment, l'action sera toujours le fruit d'une connaissance imparfaite de la situation, des règles et des motivations d'autrui. Dans un contexte de rationalité limitée (Simon et March, 1958), on parle davantage de solution satisfaisante que de solution optimale (Ramstad, 1998).

\section{Évidemment, l'action sera toujours le fruit d'une connaissance imparfaite de la situation, des règles et des motivations d'autrui}

C'est donc avec l'école institutionnalistepragmatique que l'action collective prend tout son sens. Commons y décrit l'institution comme l'action collective qui contrôle, libère et étend l'action individuelle. La définition originelle de Commons (1934: 73) est «le contrôle de l'action collective [...] qui peut à la fois restreindre, libérer ou développer l'action individuelle ». Concrètement, les acteurs sont régis par des institutions qui contrôlent leur activité, c'est-à-dire des modes de pensée et d'actions requis ou permis dans un monde du travail plus ou moins organisé ${ }^{7}$ (Gislain, 2003 :10). L'institutionnaliste est une théorie de l'action collective qui met au premier plan le rôle des institutions, «c'est-àdire les arrangements sociaux dans la stabilisation des interactions entre les hommes dans leurs activités de production, d'échange et de répartition » (Bazzoli, 1999:53). Cette approche ne relève pas d'un déterminisme complet, «il s'agit plutôt [...] de prendre analytiquement en compte la réalité de la construction sociale de l'action instituée » (Gislain, $2003: 11$ ). La conduite des sujets agissants est socialement construite selon les 
attentes et leur statut d'acteur, tels des guides comportementaux, des règles opérantes de conduite instituées et en perpétuelle évolution.

L'action collective pour Commons prend conséquemment un sens plus profond que celle donnée dans la littérature économique et sociologique où l'expression action collective sous-tend un processus délibératif interindividuel par les membres d'un collectif où l'action est décidée en commun (Morel, 1996 :140). Elle est également différente de la définition dominante issue de la discipline des relations industrielles (Freidman et Medoff, 1980 et Hirchman, 1972), laquelle fait référence à la conjugaison des efforts pour atteindre un objectif spécifique, c'est-à-dire à l'action concertée compte tenu de la similarité des intérêts. L'action collective se révèle davantage un ensemble de règles de comportement qui assujettissent les individus dans leur activité comme condition de socialisation, sous peine d'autisme social. Les interactions sociales font que les normes collectives s'intègrent et se construisent de façon réciproque. Selon George H. Mead, cette conscience particulière de l'individu, du soi, sans démarcation claire avec la totalité sociale, conduit à l'adaptation réciproque et mutuelle des conduites et des actions dans l'élaboration du processus social (1963). Ce contrôle est le fondement même des relations sociales, de ces interrelations entre les humains inscrites dans les trois dimensions que sont le conflit, l'ordre et la dépendance ${ }^{8}$ (Commons, 1934 :4). C'est l'action collective qui régule les conflits et maintient l'ordre dans un monde de rareté ${ }^{9}$, car la tendance naturelle de l'organisation sociale est davantage marquée par le conflit et le désordre ${ }^{10}$. La seule stabilité possible est celle qui est créée par l'action collective (Commons, 1934 : 213).

Pour Commons, les relations sont corrélatives et réciproques. " $\mathrm{Ce}$ contrôle des actes d'un individu résulte toujours, et a pour intention de résulter, en un bénéfice pour d'autres individus » (1934:70). Ainsi, la libération de l'action individuelle des uns s'opère au moyen de la restriction de l'action de l'autre. Mais l'action collective se révèle beaucoup plus qu'une simple restriction ou libération de l'action individuelle. Elle prolonge aussi l'action individuelle au-delà de ce que peut faire une personne en permettant d'en étendre le rayonnement. C'est l'action collective qui permet l'expansion de la volonté individuelle. L'action collective agit comme levier en mettant des moyens collectifs matériels ou immatériels qui augmentent la capacité d'actions individuelles (Morel, 1996 :157-8).

Commons accorde une grande importance à la volonté, volonté individuelle permettant de choisir entre plusieurs opportunités. Même si les règles du groupe sont un facteur explicatif du comportement individuel, cela ne veut pas dire que les individus se comporteront de façon identique. L'institutionnaliste de Commons ne peut être résumé à une théorie déterministe ou fonctionnaliste (Morel, 1996 :78). Conformément à la philosophie du pragmatisme, l'individu est un être agissant et sa volonté d'agir (willingness) se déploie dans l'action, selon la situation définie par l'action collective (ibid., 1996 : 149). Les conduites sont le plus souvent l'expression d'hypothèses habituelles sur ce qu'il est possible de faire. Elles sont guidées par les anticipations qui se forment par les interactions entre les individus au lieu de se développer à partir du point de vue d'un agent isolé, d'un stratège: la conception de l'individu est «sociologique » (Ramstad, 1993 : 185). Même si les individus sont contraints par l'action collective, l'institution, ils ne le sont pas totalement car les individus sont à l'origine de l'évolution des institutions. La volonté humaine en action est le facteur central qui façonne l'orientation de l'évolution (Commons, 1934 :629, 638). Ainsi et dans le même esprit que la conception meadienne ${ }^{11}$, «les acteurs construisent leurs rôles, leurs conduites, leurs personnalités instituées dans et par les transactions » 
(Gislain, 2003 :11). En conséquence, la théorie institutionnaliste de Commons est non seulement une théorie de l'action, mais aussi une théorie de l'évolution, dans ce monde en perpétuelle mouvance.

\section{Le comportement de l'individu ne peut être compris que par le comportement du groupe dont il fait partie}

Au risque de se répéter, dans la théorie de l'action d'inspiration pragmatiste, le comportement de l'individu ne peut être compris que par le comportement du groupe dont il fait partie et ce, dans un processus interactif et itératif d'adaptation mutuelle non déterminé et en devenir. C'est l'avenir qui détermine le comportement actuel. La représentation de l'avenir est ainsi vue comme déterminante de la conduite actuelle (Mead, 1963: 100). Pour Commons (1934: 84), «les hommes vivent dans le futur mais agissent dans le présent ». Dans ce contexte, Gislain (2006 : 244) considère que la futurité est l'un des fondements de la théorie pragmatiste institutionnaliste. Pour ce dernier (2003: 12), l'approche institutionnaliste trouve sa pertinence dans cette dimension cruciale qu'est la futurité. Ainsi,

l'inscription dans une futurité (futurity) socialement construite, dans un à-venir qui est structuré collectivement, une sorte de devenir commun plus ou moins sécurisé pour chacun et qui possède donc des dimensions beaucoup plus riches que celle du simple temps chronologique.

Enfin, les interactions, les pratiques des acteurs devraient être orientées vers un idéal pragmatique, dans une optique de raisonnabilité. Cette question incontournable du raisonnable, limitant la coercition et la subordination abusive, est au centre de l'analyse commonsienne lorsqu'il traite d'une dimension normative acceptable (Gislain, $2003: 18-19)$. Ce principe éthique permet d'orienter le processus de résolution de conflits vers un ordre social acceptable, lequel englobe les pratiques jugées préférables d'une société donnée (Commons, 1934: 683-4). Pour Commons, il importe de mettre en œuvre des réponses collectives aux problèmes créés par les conflits et l'inégalité des rapports de pouvoir au sein de la société afin d'atteindre plus d'égalité dans la répartition des charges et des bénéfices. Dans un monde de rareté, l'individu peut être freiné par le collectif dans son appropriation de la richesse compte tenu notamment de la notion d'utilité publique ; l'individu peut nuire aux autres s'il prend tout ce qu'il veut (Commons, 1934 : 225). L'action individuelle n'est plus une volonté indépendante des règles sociétales (Bazzoli, 1999: 130), ce qui fait que le contrôle de l'action collective n'est pas limité qu'à l'espace organisationnel. Pour John Dewey cité par Renault (2006 : 3), comme...
il n'existe pas d'environnement «pur» détaché du sujet, mais que l'environnement est médiatisé par la perception qu'en ont les sujets, perception qui est elle-même déterminée par des facteurs sociaux, culturels, affectifs [...] le pragmatisme dénonce la séparation entre l'organisme et l'environnement, entre l'économie et la société, entre l'entreprise et la société [...]. [C]omme l'avait souligné Dewey, l'organisme et l'environnement ne sont pas séparés, l'un et l'autre se co-déterminent et ne se définissent qu'en relation ${ }^{12}$.

En conséquence, un individu ne peut jamais être étudié comme un être isolé. Il doit donc être appréhendé comme un être social dont l'action individuelle relève des coutumes de ses groupes sociaux d'appartenance et des règles de conduite (règles opérantes) du collectif dont il est membre (Morel, 1996 :142). Le même raisonnement s'applique également à l'organisation qui n'est pas une entité détachée de sa société d'appartenance. Dans la perspective commonsienne, l'action collective dépasse le cadre organisationnel. 
En terme épistémologique, le pragmatisme philosophique, en tant que vérité et réalité raisonnable, rompt avec le dualisme cartésien de la pensée et de l'action qui se caractérise notamment par l'opposition entre l'inductif et le déductif ${ }^{13}$, pour adopter un processus de production de connaissances axé sur l'activité humaine et l'expérimentation (Bazzoli, 2000, Gislain, 2004, 2003). Comme le précise Renaud (2006 : 124), « pour le pragmatisme, la connaissance est issue d'expériences, de problèmes résolus, d'essais, d'erreurs [...] elle n'est donc jamais séparée de l'action ». Ce procédé que Pierce appelle «abduction» et Dewey «enquête sociale » est une démarche d'expérimentation active ou de mise à l'épreuve qui repose sur des hypothèses de travail provisoires, retenues comme référence opératoire, qui sont mises en relation avec la réalité $^{14}$ (Bazzoli, 2000, Bazzoli et Dutraive, 2006 et Gislain, 2006). En d'autres termes, ce sont des outils mentaux d'investigation construits et modifiés par l'expérience ou, comme le soulignent Bazzoli et Dutraive (2006:20) : «une théorisation produite de l'interaction permanente entre l'expérience des faits et la pensée des faits ». Ces outils d'investigation, s'assimilant aux «formules » idéales-types au sens weberien d'un outil analytique de compréhension ${ }^{15}$, et réellestypes, « c'est-à-dire saisissant la réalité observable »(Gislain, $2003: 26)$, permettent une inférence expérientielle à partir de laquelle l'action collective réelle sera démontrée. En clair, l'approche implique une méthodologie d'observation du tout en action dans son contexte, de la relation des parties au tout et non réductible à chacun des acteurs pris isolément (Bazzoli et Dutraive, 2006, Ramstad, 1986).

Comme outil de recherche, l'étude de cas s'y révèle largement adaptée, car elle permet notamment d'investiguer un phénomène contemporain dans un contexte de vie réel (Yin, 1984: 23). Selon Jacques Hamel (1997 : 34), suivant la tradition de George H.
Mead (psychologie sociale), lui-même influencé par la philosophie pragmatiste de Dewey, «la vie sociale, définie comme un processus ou un mouvement, ne peut être saisie qu'à condition de s'y insérer et de comprendre les significations qui lui sont attribuées par ses propres acteurs ». La proximité avec la réalité que permet l'étude de cas est, selon Bent Flyvbjerg (2004: 422, 429), un préalable pour faire avancer la compréhension. Malgré 1'étiquette descriptive communément associée à l'étude de cas, la vérification théorique peut aussi s'y appliquer (Flyvbjerg, 2004, Hamel, 1997). L'étude de cas, comme toute autre étude, est sujette à ce que l'on vérifie la valeur opératoire des catégories formulées et des procédés par lesquels elle s'articule. Si elle se forme et se règle rigoureusement, comme dans l'œuvre entière de Commons ${ }^{16}$, sa démonstration est possible et ouverte à son utilisation dans un contexte de vérification conceptuelle et de reformulation, le cas échéant, en tenant compte du réel. Selon Hamel (1997 : 115),

\section{l'étude de cas met donc en jeu une théorie descriptive dont l'épistémologie nous rappelle qu'elle est le prélude à une théorie explicative, c'est-à-dire une théorie marquée par l'idée d'opérations explicitement et univoquement définies et réglées dans l'intention d'expliquer.}

Enfin, en étude de cas, l'observation in situ (en situation et en temps réels) et les entretiens ouverts s'y taillent une place de choix comme outils de cueillette (Hamel, 1997).

Dans ce cadre et comme méthode de cueillette, l'ethnographie possède l'indéniable avantage de ne pas séparer la recherche de l'action (Foote Whyte, 1984 : 20; Katz, 2002). Les observations sont réalisées dans un contexte de travail et d'interaction entre personnes. L'ethnographie a joué un rôle important dans

L'ethnographie possède l'indéniable avantage de ne pas séparer la recherche de l'action 
l'étude du travail, notamment en ce qui concerne l'observation de la négociation collective (Friedman, 2004). En outre, elle permet de décrire comment les individus perçoivent leurs propres actions et celles des autres, et dans quel contexte l'action prend place, notamment en pénétrant l'antre de la présumée cause et du présumé effet (Friedman et McDaniel, 1998 : 116-117). Plus important encore, elle permet la compréhension de ce qui

\section{Conclusion}

Fait particulièrement risible, nous ne pouvons passer sous silence la contribution largement ignorée de J.R. Commons comme pionnier de la discipline de la gestion des ressources humaines (Kaufman, 1998 ${ }^{18}$ ). En effet, il n'existe aucune mention du rôle de l'économiste institutionnaliste qu'est Commons, dans la littérature traitant de la naissance et de l'historique de la gestion des ressources humaines.

En voici un bref historique. Au début des années 20, l'économie était la discipline sur laquelle s'articulaient les autres sujets en administration tels la finance et la gestion de personnel. La gestion des ressources humaines dans les universités tendait à être séparée en deux groupes: l'un relatif à l'économie du travail, plus «social» et sympathique à la cause syndicale, avait pour cible l'influence de l'environnement externe (l'économique, le légal, le social et le politique) comme déterminant des pratiques et des résultats en gestion des ressources humaines. L'autre, combinant le management à l'administration, la psychologie industrielle et la performance organisationnelle, portait davantage son attention sur le rôle interne de ces différentes dimensions. Cette perspective reçut davantage de visibilité que la première associée à Commons. Néanmoins, son impact est manifeste lors de la création en 1920 à l'Université du Wisconsin, de l'une des est difficilement visible et inhérent au collectif, c'est-à-dire les normes exprimées et renforcées par l'interaction sociale (ibid.: 119). Cela cadre parfaitement avec la perspective institutionnaliste pour laquelle «les jugements et les actions [de l'individu comme acteur social] sont un produit émergent de l'interaction avec l'autre ${ }^{17}$ » (Tool, 1994 : 211).

premières séries de cours aux États-Unis en gestion des ressources humaines.

Dans ses ouvrages Industrial Goodwill et Industrial Gouvernement parus respectivement en 1919 et 1920, Commons jette un regard éclairant sur cette discipline émergente et analyse par une série d'études de cas aux États-Unis, différentes pratiques en gestion des ressources humaines (Kaufman, 1998 :335). Commons ne portait pas beaucoup d'attention sur le comment faire (how to), mais s'appuyait davantage sur une perspective globale en analysant les différentes approches en management et les origines philosophiques sous-jacentes au labor afin d'y résoudre les problèmes d'emploi pour les entreprises et la nation; par exemple, en décrivant, dans les secteurs publics et privés, l'importance de l'investissement en éducation et en formation, en soulignant l'importance de la sécurité d'emploi comme facteur de motivation, etc. Sans s'étendre davantage sur son apport, soulignons brièvement en terminant la création de cinq modèles relatifs à la gestion des ressources humaines qui sont encore largement d'actualité : 1) la ressource humaine comme une marchandise (commodity), 2) la gestion des ressources humaines largement inspirée de l'ingénierie à saveur taylorienne, 3) l'investissement ressource humaine comme élément d'utilité publique, 4) le modèle des relations du travail et la création de conditions 
psychologiques de bonne volonté (goodwill) (confiance, non-discrimination, transaction juste, etc.) nécessaires à l'efficacité organisationnelle et enfin, 5) le modèle citoyen, décrivant la démocratie industrielle et la prise de parole des salariés dans la détermination des conditions de travail.
Dans une optique de causalité circulaire, terme caractéristique de la démarche méthodologique originale de Commons, il est, croyons-nous, venu le temps de réhabiliter la richesse et la justesse de son cadre analytique en regard de la gestion des ressources humaines et du management.

\section{Notes et références}

1 C'est principalement à partir des travaux d'Elton Mayo à l'usine de Hawthorne de la Western Electric que l'on découvrit le «facteur humain » où, pour la première fois, des éléments d'ordre affectif et émotif étaient démontrés déterminants pour la productivité (Aktouf, 1994). Fait important à souligner, les travaux précurseurs de cette école, le fameux «effet Hawthorne», ont tout de même servi à montrer concrètement, et pour la première fois, l'effet du groupe sur le comportement de l'individu (Bernoux, 1990).

2 Pour Sylvie Morel (2004 : 5), «conformément à l'individualisme méthodologique, la société est présentée comme étant une collection des individus autonomes et indépendants, entrant en relation les uns avec les autres par le biais des échanges marchands. Est ainsi mise de l'avant une conception du lien social comme unique lien marchand ».

3 Un système, c'est un ensemble d'éléments interdépendants, c'est-à-dire liés entre eux par des relations de telle sorte que si l'une est modifiée, les autres le sont aussi et que, par conséquent, tout l'ensemble est transformé (Bertalanffy, 1975). Cependant, cette perspective ne peut avoir la prétention d'être une théorie. Elle est davantage une description de l'organisation et de ses différentes fonctionnalités.

4 Veblen est économiste de la fin $19^{\mathrm{e}}$ et du début du $20^{\mathrm{e}}$, et est considérée comme précurseur, avec Commons, de l'institutionnaliste économique.

5 À bien y penser, dans la description systémique évoquée précédemment, la référence à la biologie est implicite, tel un corps humain fait de différents organes liés entre eux par le réseau sanguin, qui est soumis aux pressions de l'environnement (maladie, humidité, température, etc.) auquel il doit s'adapter.

6 La transaction, concept fondamental de Commons, considérée comme la plus petite unité de l'économie institutionnaliste, est notamment appréhendée comme un processus commun de création de règles collectives pour gérer les conflits et organiser les relations (Commons, 1934). Les interactions dans la transaction « [...] sont des situations de négociation où les volontés humaines particulières se confrontent et ajustent les règles à la situation » (Bazzoli, $2000: 15)$. Elle prend donc une tout autre forme que celle rattachée à l'économie orthodoxe ou traditionnelle dans sa forme pure marchande, c'est-à-dire centrée sur l'échange et le modèle du marché concurrentiel parfait (Bazzoli, 2000, Morel, 2004).

7 Pour Commons, il y a les règles organisées que sont les règles opérantes de conduite et celles non organisées que sont les habitudes individuelles et les coutumes.

8 Par exemple, dans chaque transaction économique, il y a conflit d'intérêt compte tenu que chaque participant tente d'obtenir le plus et de donner le moins. De même, personne ne peut vivre ou prospérer seul. La subsistance individuelle est inévitablement liée à la relation de dépendance avec autrui, à l'interdépendance première entre individus (Commons, $1934: 118$ ).

9 Pour chacun, les besoins sont supérieurs aux disponibilités en biens et services.

10 A noter que la conception orthodoxe de l'économie, qui présuppose l'harmonie naturelle des intérêts inspirée de la raison divine, considère la loi et le conflit d'intérêts comme des éléments pathogènes (Commons, 1934 : 120).

11 Même si Commons et Mead ne se sont pas cités mutuellement, leurs oeuvres datant de la même époque, il est démontré que leurs postures respectives sont largement similaires. Voir à cet égard ALBERT, A. et RAMSTAD, Y., 1998, «The Social Psychological Underpinnings of Commons's Institutional Economics II : The Concordance of George Herbert Mead's "Social Self" and John R. Commons "Will", dans Journal of Economics Issues, vol. XXXII, $\mathrm{n}^{\circ} 1$, mars, p. 1-46.

12 George Herbert Mead (1963 : 109) traduisit également plus tard cette thèse en soulignant que «l'organisme et le milieu se déterminent réciproquement et sont mutuellement dépendants ». 
13 Cette posture n'est ni totalement déductive, c'est-à-dire assimilable à une vérification stricte d'hypothèses déduites par le chercheur et ni totalement inductive, telle la méthode de théorisation ancrée élaborée par Glaser et Strauss (1967) qui a pour objectif de construire une théorie fondée qu'à partir des phénomènes sociaux.

14 C'est sur la base de ce procédé que «Commons a mis en œuvre [son] monde original de théorisation » (Bazzoli, $2006: 9)$.

15 Selon Weber (1995), les idéaux-types sont en quelque sorte une construction logique «pure » basée sur des possibilités objectives qui permettent de comprendre, par comparaison, le déroulement effectif et réel de l'activité sociale. Les idéaux-types sont utilisés pour comprendre le «sens visé » ou, dit autrement, la logique spécifique de l'acteur.

16 Selon Jean-Jacques Gislain (2003 : 26), l'étude de cas fut largement contributoire au prestige scientifique de Commons auprès des praticiens en relations industrielles.

17 Pour reprendre l'expression de Commons « human will in action ».

18 Cette conclusion est presque entièrement inspirée du texte de Kaufman. KAUFMAN, Bruce E., (1998), « John R. Commons : His Contributions to the Founding and Early Development of the Field of Personnel/HRM », dans Proceeding of the Fiftieth Annual Meeting, Chicago, Industrial Relations Research Associations Series (IRRA), vol. 1, éditrice Paula V. Boos, Chicago, p. 328-340.

\section{Bibliographie}

AKTOUF, O., 1994, « De Elton Mayo aux sciences du comportement organisationnel », dans le Le management entre tradition et renouvellement, $3^{\mathrm{e}}$ édition, Montréal, Gaëtan Morin éditeur, p. 201-230.

AKTOUF, O., 2006, Le management entre tradition et renouvellement, 4 e édition, Montréal, Gaëtan Morin éditeur, 663 p.

ALBERT, A. et RAMSTAD, Y., 1998, « The Social Psychological Underpinnings of Commons's Institutional Economics II : The Concordance of George Herbert Mead's "Social Self" and John R. Commons "Will", dans Journal of Economics Issues, vol. XXXII, $n^{\circ} 1$, mars, p. 1-46.

AMBLARD, H., BERNOUX, P. et al., 2005, Les nouvelles approches sociologiques des organisations, Éditions du Seuil, $3^{\mathrm{e}}$ augmentée, Paris, 292 p.

BAZZOLI, Laure, 2000, «L'économie institutionnaliste du travail de J.R. Commons : un "pragmatisme en action" », dans Cahier du Gratice, $\mathrm{n}^{\circ} 19$.

BAZZOLI, Laure, 1999, L'économie politique de John R. Commons : Essai sur l'institutionnalisme en sciences sociales, Éditions L'Harmattan, Paris, 234 p.

BAZZOLI, Laure et DUTRAIVE, Véronique, 2006, «Fondements pragmatistes de l'institutionnalisme en économie. Théorie de la connaissance et théorie de l'action chez Veblen et Commons », dans Revue de philosophie économique, $\mathrm{n}^{\circ} 13 \mathrm{p}$.

BECKER, H.S., 2002, Les ficelles du métier : comment conduire sa recherche en sciences sociales, Éditions La Découverte, Paris, $353 \mathrm{p}$.

BERNOUX, P., 1990, La sociologie des organisations, Éditions du Seuil, $3^{\mathrm{e}}$ édition, Paris, 378 p.

BERNOUX, P., 2004, Sociologie du changement dans les entreprises et les organisations, Paris, Seuil, 307 p.

BERTALANFFY, L.V., 1975, Perspectives on general system theory : Scientific-philosophical studies, Edgar Taschdjian, (éditeur), $183 \mathrm{p}$.

BOURDIEU, P., 2004, Raisons pratiques sur la théorie de l'action, Les Éditions du Seuil, Paris, $2^{\mathrm{e}}$ édition, 246 p.

COLlERETTE, P., DELISLE, G. et al., 2002, Le changement organisationnel : théorie et pratique, Presses de l'Université du Québec, Québec, 173 p.

COMMONS, J. R., 1934, Institutional Economics : Its Place in Political Economy, The University of Wisconsin Press, Madison, édition 1959, Vol. 1 et 2, 921 p.

CROZIER, M. et FRIEDBERG, E., 1981, L'acteur et le système, Les éditions du Seuil, Paris, 2e édition, 500 p.

DONNADIEU, G., 2005, «La régulation sociale », dans Les ressources humaines, Ouvrage conçu et coordonné par Dimitri Weiss, Édition d'organisations, $2^{\mathrm{e}}$ édition, Paris, p. 179-235.

FLYVBJERG, Bent, 2004, "Five misunderstandings about case-study research », dans Qualitative Research Practice, Clive Seale, Giampietro Gobo, Jaber F. Gubrium, and David Silverman éditeurs, London Thousand Oaks, CA : Sage, p. $420-434$.

FOOTE WHITE, William, 1984, Learning from the field : a guide from experience, Sage publications, Beverly Hills, Ca, $295 \mathrm{p}$.

FRIEDMAN, Ray, 2004, «Studying Negociations in Context : An Ethnographic Approach », dans International Negotiation, 9, p. 375-284.

FRIEDMAN, R.A. et McDANIEL, D. C., 1998, «In the Eye of the Beholder: Ethnography in Study of Work », dans Researching the World of Work: Strategies and Methods in Studying Industrial Relations, ILR Press, sous la direction de Keith Whitfield et George Strauss, p. 113-126.

FREEMAN, R.B. et MEDOFF, J.L., 1980, « Le syndicalisme à deux visages », Revue économique, no 3, mai, p. $505-538$.

GISLAIN, J.-J., 2006, « Le processus d'innovation sociale : un cadre d'analyse institutionnaliste pragmatiste », dans Innovations sociales dans le travail et l'emploi : recherches empiriques et perspectives théoriques, sous la direction de P.A, Lapointe et G. Bellemare, Les Presses de l'Université Laval, p. 237-274. 
GISLAIN, J.-J., 2004, «Futurité et toposité : situlogie des perspectives d'action », dans Géographie, économie et société, $\mathrm{n}^{\circ}$ 6., p. 209-213.

GISLAIN, J.-J., 2003, « L'institution des relations industrielles : le cadre analytique de J.R. Commons, ${ }^{\mathrm{o}} 2$ 2, $2^{\mathrm{e}}$ semestre, p. 9-56. GISLAIN, J.-J. et STEINER, P., 1995, La sociologie économique 1890-1920, Paris, Presses Universitaires de France, 235 p. GLASER, B.G. et STRAUSS, A.L., 1967, The Discovery of Grounded Theory, Chicago, Aldine.

HAMEL, J., 1997, Étude de cas et sciences sociales, L'Harmattan, Montréal, 123 p.

HAMMER, M. et CHAMPY, J., 1993, Le reengineering, Paris, Dunod, 247 p.

HIRSCHMAN, A.-O., 1972. Face au déclin des entreprises et des institutions, Économie et Humanisme, Les éditions ouvrières, Paris, $141 \mathrm{p}$.

JACOB, R., 1992, Flexibilité organisationnelle et gestion des ressources humaines, GREPME, Cahier de recherche, 19 p.

JOAS, Hans, 1999, La créativité de l'agir, Les éditions du Cerf, Paris, 306 p.

KATZ, Jack, 2002, «From how to why: on luminous description and causal inference in ethnography (part 2)», dans Ethnography, Sage publications, vol. 3 (1), p. 63-90.

KAUFMAN, B. E., (1998), «John R. Commons : His Contributions to the Founding and Early Development of the Field of Personnel/HRM », dans Proceeding of the Fiftieth Annual Meeting, Chicago, Industrial Relations Research Associations Series (IRRA), vol. 1, éditrice Paula V. Boos, Chicago, p. 328-340.

LAPOINTE, P.A., 1995, «La réorganisation du travail : continuité, rupture et diversité », dans La réorganisation du travail : efficacité et implication, $50^{\mathrm{e}}$ Congrès des relations industrielles, Sainte-Foy, PUL, p. 3-43

LORINO, P., 2007, « Un défi pour les sciences de la gestion : le tournant paradigmatique du modèle de la décision au modèle de l'action collective " dans Science du management : épistémique, pragmatique et éthique, coordination Alain Charles Martinet, Vuibert, Paris, p. 69-83.

MARCH, J.G., 1981, «Footnotes to Organizational Change », dans Administrative Science Quarterly, n 26, p. $563-577$.

MARCH, J.G. et SIMON, H. A., 1958, Organisations, Wiley, New York, 262 p.

MEAD, G.H., 1963, L'esprit, le soi et la société, Presses Universitaires de France, Paris, version originale 1934, 332 p.

MINTZBERG, H., 1994, « The Fall and Rise of Strategic Planning » dans Harvard Business Review, p. 107-114.

MOREL, S., 1996, Le workfare et l'insertion : une application de la théorie institutionnaliste de John R. Commons, Thèse de doctorat en science économique, Université de Paris 1, sous la supervision de Bernard Gazier, 668 p.

MOREL, S. 2004, Citoyenneté, travail et emploi : les éclairages de la théorie économique et de l'analyse de genre, Texte de la communication présentée au colloque intitulé La citoyenneté au travail ? Réflexions sur le milieu de travail de l'avenir, du Centre de recherche interuniversitaire sur la mondialisation et le travail (CRIMT), Université Laval, 21-23 juin.

PARSONS, T., 1937, The Structure of Social Action, New York.

RAMSTAD, Y., 1986, «A Pragmatist's Quest for Holistic Knowledge : The Scientific Methodology of John R. Commons », dans Journal of economics issues, vol. XX, $\mathrm{n}^{\circ}$ 4, Décembre, p. 1067-1105.

RAMSTAD, Y., 1994, «On The Nature of Economic Evolution : John R. Commons and the Metaphor of Artificial Selection », dans Evolutionary and Neo-Schumpeterian Approaches to Economics, édité par Lars Magnusson, Boston/Dorrecht/Lancaster : Kluwer-Nuhoff Publishing, p. 65-121.

RAMSTAD, Y., 1998, « Common's Institutional Economics : A Fondation of Industrial Relations Theory? », dans Proceeding of the Fiftieth Annual Meeting, Chicago, Industrial Relations Research Associations Series (IRRA), vol. 1, éditrice Paula V. Boos, Chicago, p. 308-319.

RAMSTAD, Y., 1993, «Institutional Economics and the Dual Labor Market Theory », dans Institutional Economics : Theory, Method, Policy, Édité par M.R. Tool, Boston/Dordrecht/London, Kluer Academic Publishers, p. 173-233.

RENAUD, Michel, 2006, «Délibération, action et démocratie : une perspective pragmatique-institutionaliste », dans La démocratie au péril de l'économie, édité par Marc Humbert et Alain Caillé, Presses Universitaires de Rennes, Rennes, p. 123-140. ROY, M., 1999, «Les équipes semi-autonomes au Québec et la transformation des organisations », dans Gestion, vol. 24, n 3, p. $76-85$.

TOOL, M.R., 1994, «An Institutionalist Mode of Inquiry », dans P.A. Klein (éd.), The Role of Economic Theory, Dordricht, Kluwer, p. 197-227.

WEBER, M., 1995, Économie et société, tome 1 : Les catégories de la sociologie, Paris, Plon/Agora, traduction Julien Freund, $411 \mathrm{p}$.

YIN, Robert K., 1984, Case Study Research : Design and Methods, Sage Publications, 160 p. 


\section{Publicité}

Ministère 